\title{
New Surface-Enhanced Raman Sensing Chip Designed for On-Site Detection of Active Ricin in Complex Matrices Based on Specific Depurination
}

Ji-jun Tang, ${ }^{\dagger, \ddagger}$ Jie-fang Sun, ${ }^{\dagger, \S}$ Rui Lui, ${ }^{\S}$ Zong-mian Zhang, ${ }^{\S}$ Jing-fu Liu, ${ }^{*, \S, \|}$ and Jian-wei Xie ${ }^{*, \$}$

${ }^{\ddagger}$ State Key Laboratory of Toxicology and Medical Countermeasures, and Laboratory of Toxicant Analysis, Institute of Pharmacology and Toxicology, Academy of Military Medical Sciences, Beijing 100850, People's Republic of China

${ }^{\S}$ State Key Laboratory of Environmental Chemistry and Ecotoxicology, Research Center for Eco-Environmental Sciences, Chinese Academy of Sciences, Beijing 100085, People's Republic of China

"Institute of Environment and Health, Jianghan University, Wuhan, Hubei 430056, People’s Republic of China

\section{Supporting Information}
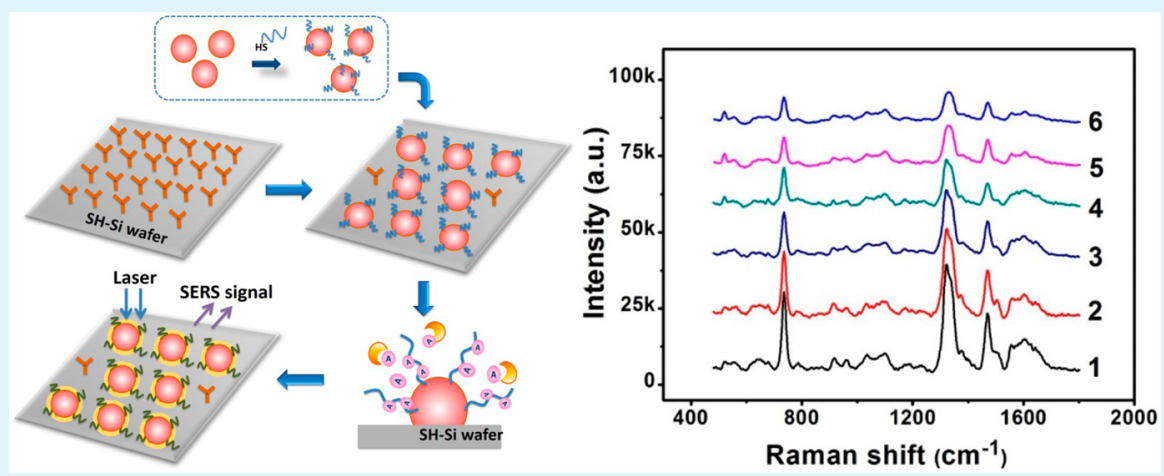

ABSTRACT: Quick and accurate on-site detection of active ricin has very important realistic significance in view of national security and defense. In this paper, optimized single-stranded oligodeoxynucleotides named poly (21dA), which function as a depurination substrate of active ricin, were screened and chemically attached on gold nanoparticles (AuNPs, $\sim 100 \mathrm{~nm}$ ) via the $\mathrm{Au}-\mathrm{S}$ bond $[\operatorname{poly}(21 \mathrm{dA})-\mathrm{AuNPs}]$. Subsequently, poly(21dA)-AuNPs were assembled on a dihydrogen lipoic-acid-modified Si wafer $(\mathrm{SH}-\mathrm{Si}$ ), thus forming the specific surface-enhanced Raman spectroscopy (SERS) chip [poly(21dA)-AuNPs@SH-Si] for depurination of active ricin. Under optimized conditions, active ricin could specifically hydrolyze multiple adenines from poly $(21 \mathrm{dA})$ on the chip. This depurination-induced composition change could be conveniently monitored by measuring the distinct attenuation of the SERS signature corresponding to adenine. To improve sensitivity of this method, a silver nanoshell was deposited on post-reacted poly $(21 \mathrm{dA})-$ AuNPs, which lowered the limit of detection to $8.9 \mathrm{ng} \mathrm{mL} \mathrm{m}^{-1}$. The utility of this wellcontrolled SERS chip was successfully demonstrated in food and biological matrices spiked with different concentrations of active ricin, thus showing to be very promising assay for reliable and rapid on-site detection of active ricin.

KEYWORDS: gold nanoparticles, surface-enhanced Raman spectroscopy, active ricin, depurination, on-site detection

\section{INTRODUCTION}

Ricin is a highly lethal toxin of approximately $64 \mathrm{kDa}$ derived from the castor bean plant. ${ }^{1}$ This protein has been intensively investigated for possible applications as an immunotoxin in cancer chemotherapy, profiting from its high lethality, wide availability, and low-cost production. With its features mentioned above plus ease of use, lack of effective antidotes, and good stability after dissemination, ricin has been considered as an ideal biological warfare agent. Given its notorious history as a toxic bioweapon, ricin remains the only protein listed as a Schedule 1 compound by the Chemical Weapons Convention. Its ever increasing threat to national security and public safety has led to urgent demand for the development of highly efficient analytical tools, which are helpful to improve biodefense and ensure the survival of affected individuals.

For the detection of ricin, multiple analysis platforms have been developed, which mainly comprise immunoassays [such as enzyme-linked immunosorbent assays (ELISAs)], instrumental analysis (such as mass spectrometry), ${ }^{3,4}$ and aptameric techniques. $^{5-7}$ When the advantages of these methods are combined, qualitative and quantitative determination of ricin can be accomplished; however, these methods fall short in

Received: December 31, 2015

Accepted: December 31, 2015

Published: December 31, 2015 
terms of risk assessment, because they are incapable of judging activity (and, therefore, toxicity) of ricin.

The test of active ricin is critical because inactivation eliminates its toxic threat. Previous studies have established some cell-based assays for active ricin; however, these methods are not practical to use in the field assay. ${ }^{8,9}$ To efficiently and promptly assess potentially hazardous situations, timely and reliable detection of active ricin in complex matrices is crucial for the first responders and military personnel. On the basis of the foregoing research, a range of analytical techniques have been developed to detect active ricin. The core of these methods is the depurination of active ricin from ribosomal RNA (rRNA) or synthetic oligonucleotide substrates (RNA or DNA), and then the toxicity of ricin is determined indirectly by measuring released adenine with various analytical tools. ${ }^{10-12}$ Although most of these methods show excellent performance, they are constrained by disadvantages, such as multi-step sample preparation, tedious covalent labeling processes, and complex chromatography/mass spectrum operation. More importantly, the strict requirements for laboratory facilities, lack of portability, and large reagent consumption would significantly limit these methods used on the battlefield, where such evaluation is the most needed. Accordingly, developing a reliable method for field rapid analysis of such a biological warfare agent in the complex matrices is a worthy goal, which would significantly impact exposure surveillance and outbreak prevention efforts.

In the last 15 years, the investigations on nanomaterial-driven analysis and spectroscopy techniques have expanded tremendously. Surface-enhanced Raman scattering (SERS) is the best positioned among all measurement techniques, representing an attractive analytical tool for environmental/biological inspection, ${ }^{13-15}$ public/food safety, ${ }^{16,17}$ and homeland security. ${ }^{18-20}$ Especially after conjunction with commercially available portable Raman instrumentation, SERS allows for the performance of accurate, non-invasive, rapid, and sensitive on-site detection.

To fabricate a specific SERS chip for active ricin detection, several single-strand oligodeoxynucleotides (ssODNs) were first evaluated as depurination substrates of active ricin under in vitro conditions, and the optimal ssODN would be attached on gold nanoparticles (AuNPs) to give ssODN-AuNPs. Subsequently, we adopted the chemical method proposed by Algar et al. $^{21}$ to attach high-density dihydrogen lipoic acid (DHLA) moieties on a $\mathrm{Si}$ wafer. The modified $\mathrm{Si}$ substrate $(\mathrm{SH}-\mathrm{Si})$ could directly anchor ssODN-AuNPs in a quasi-periodic array based on the multidentate $\mathrm{Au}-\mathrm{S}$ binding, thus obtaining a mechanically stable SERS chip (ssODN-AuNPs@SH-Si). The SERS chip retains an easily accessible open surface topology, where the detector module, i.e., ssODN-AuNPs, can specifically react with active ricin and induce adenine release from the sensing interface. This composition change would lead to distinctive SERS signal attenuation based on the fingerprint of adenine and offer significant promise for active ricin detection. To obtain an amplified SERS signal, a silver nanoshell was grown on the surface of post-reacted ssODNAuNPs@SH-Si via in situ solution phase deposition. When the advantage of this additional step is taken, the chance to squeeze the reporter ssODN present right at the "hot spot" of metal nanoassembly is greatly increased, which offers a remarkably improved SERS signal for ssODN. ${ }^{22}$ On the basis of this protocol, a simple and reproducible SERS chip was developed for highly sensitive and selective screening of active ricin in complex matrices. The fabricating process of the SERS chip and its application in sensing active ricin are illustrated in Scheme 1.

Scheme 1. Fabrication of the SERS Chip and Its Detection for Active Ricin ${ }^{a}$

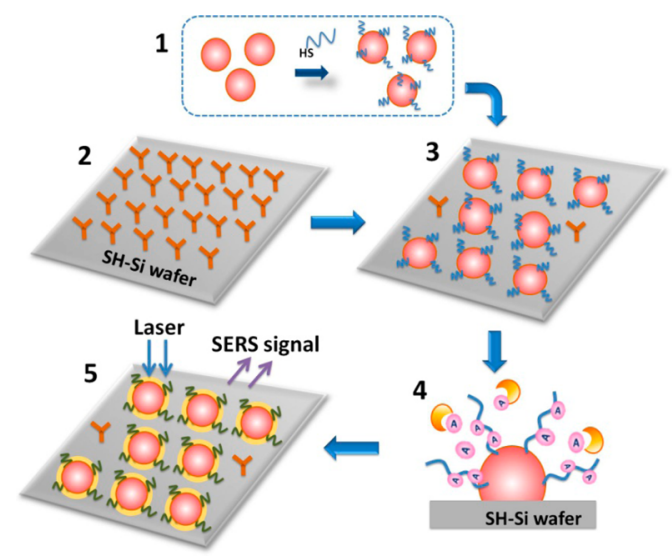

(1) Preparation of ssODN-AuNPs. (2) Modification of the Si wafer with multiple DHLA moieties. (3) Self-assembly of ssODN-AuNPs on $\mathrm{SH}-\mathrm{Si}$. (4) Specific active ricin depurination process on the interface of the SERS chip. (5) Signal collection after Ag nanoshell deposition on the post-reacted SERS chip.

\section{EXPERIMENTAL SECTION}

2.1. Safety Considerations. Because ricin is an extremely toxic protein, which is listed as a Schedule 1 compound of the Chemical Weapons Convention, all related experiments should strictly follow the operating safety guidelines of the laboratory.

2.2. Materials and Chemicals. Intact ricin with the purity exceeding $95 \%$ by sodium dodecyl sulfate-polyacrylamide gel electrophoresis (SDS-PAGE) analysis was obtained from castor beans according to the literature. ${ }^{23}$ All ssODNs were synthesized and purified by the supplier (Sangon Biological Engineering Technology \& Services Company, China). (3-Aminopropyl)triethoxysilane (APTMS), N,N'-dicyclohexylcarbodiimide (DCC, 99\%), 6-mercaptohexanol (MHO), tris(2-carboxyethyl)phosphine (TCEP), lipoic acid (LA), lysozyme, pronase, trypsin, PNGase $\mathrm{F}$, and $\beta$-glucuronidase $(\beta$ GUS) were purchased from Sigma-Aldrich, Inc. (St. Louis, MO). Desalting illustra NAP-5 column was the product of GE Healthcare. All other reagents were of analytical grade or better. Deionized water purified by a Milli- $Q$ water purification system (Millipore, MA) was employed throughout the experiments.

2.3. Evaluation of the Active Ricin Depurination Substrate and Its Immobilization on AuNPs. Different sizes of citratestabilized AuNPs $(\sim 60, \sim 75, \sim 90$, and $\sim 100 \mathrm{~nm})$ were prepared according to the kinetically controlled seeded growth method. ${ }^{24}$ Their morphologies were characterized using transmission electron microscopy (TEM, JEOL model 1200EX) operated at an accelerating voltage of $100 \mathrm{kV}$. The extinction spectra of AuNPs was collected by a Shimadzu 3600 ultraviolet-visible-near infrared (UV-vis-NIR) spectrometer. A variety of synthetic ssODNs (their structures are listed in Figure S1 and Table S1 of the Supporting Information) were evaluated to select the optimal depurination substrate. To construct the SERS chip for active ricin detection, the following sequences was used: 5'-SH-AAAAAAAAAAAAAAAAAAAAA [referred to herein as 5 '-SH-poly (21dA)], which was conjugated to different sized citrateAuNPs $(\sim 60, \sim 75, \sim 90$, and $\sim 100 \mathrm{~nm})$ based on the literaturereported method. ${ }^{25}$ Detailed experimental procedures were listed in the Supporting Information.

2.4. Fabrication of the Specific SERS Chip. Briefly, a cleaned and dried Si wafer, trimmed to an area of $3.0 \times 3.0 \mathrm{~mm}^{2}$, was modified with APTMS to derive the amine group on its surface, which was then 
linked with multiple LA ligands via DCC-mediated chemical crosslinking (see the Supporting Information). ${ }^{21}$ The LA-modified Si wafers (LA-Si) were immersed in a $50 \mathrm{mmol} \mathrm{L}^{-1}$ TCEP aqueous solution $\left(10 \mathrm{mmol} \mathrm{L}^{-1}\right.$ Tris $-\mathrm{HCl}$ at $\left.\mathrm{pH} 7.4\right)$ at room temperature for a minimum of $4 \mathrm{~h}$ to reduce the disulfide bond of LA and generate dihydrogenlipoic acid (DHLA), thus obtaining multidentate thiolappending groups on the $\mathrm{Si}$ wafer $(\mathrm{SH}-\mathrm{Si})$. The modification process for the $\mathrm{Si}$ wafer and its characterization by $\mathrm{X}$-ray photoelectron spectroscopy (XPS) were given in the Supporting Information. SH-Si was then rinsed with water and immediately immersed into the poly $(21 \mathrm{dA})-$ AuNP solution $\left(10 \mathrm{nmol} \mathrm{L} \mathrm{L}^{-1}\right)$ which had not been treated by $\mathrm{MHO}$, for their assembly onto $\mathrm{SH}-\mathrm{Si}$ via strong $\mathrm{Au}-\mathrm{S}$ binding. Following incubation of $12 \mathrm{~h}$ at room temperature, poly(21dA)-AuNPs@SH-Si was washed with water to remove non- or weakly adsorbed AuNPs. Subsequently, ligand exchange was performed by immersing obtained poly(21dA)-AuNPs@SH-Si in MHO $\left.(0.2 \mathrm{~mL} \text { of } 1.0 \mu \mathrm{mol} \mathrm{L})^{-1}\right)$ for $5 \mathrm{~min}$ and then washing sequentially with ethyl acetate, methanol, and copious ultrapure water. On the basis of the same procedures, poly (21dA)-AuNPs of different Au core sizes $(\sim 60, \sim 75, \sim 90$, and $\sim 100 \mathrm{~nm})$ were assembled on $\mathrm{SH}-\mathrm{Si}$. Besides, the control sample of the MHO-modified AuNPs $(\sim 100 \mathrm{~nm})$ array on SH-Si (MHO-AuNPs@SH-Si) was also prepared (see the Supporting Information). Finally, the SERS chip was observed by a field-emission scanning electron microscopy (FESEM, Hitachi SU-8020) to confirm the distribution of AuNPs. The collection of SERS spectra and Raman mapping was performed on a Renishaw InVia Raman microscope. The detailed experiments, Raman measurement conditions, and the signal processing method were given in the Supporting Information.

2.5. Sensing Procedures by the SERS Chip for Active Ricin. The detection systems containing poly(21dA)-AuNPs@SH-Si and various concentrations of the active ricin in ammonium acetate buffer $\left(50 \mu \mathrm{L}, 10 \mathrm{mmol} \mathrm{L}^{-1}, \mathrm{pH} 4.0\right)$ were reacted in a 96-well plate. After incubation at $38{ }^{\circ} \mathrm{C}$ with gentle shaking, the reaction was terminated by discarding the supernatant, washing with Tris buffer, and then drying under a stream of $\mathrm{N}_{2}$ for immediate Raman measurement. To monitor the kinetic of the depurination by active ricin on poly(21dA)-AuNPs@SH-Si, the SERS signals of the chips were collected at $0,15,30,45,60,75,90,105$, and $120 \mathrm{~min}$, respectively. To test the specificity of the established assay, some other proteins were tested separately with the same procedures as that of active ricin. To improve the sensitivity, post-reacted poly(21dA)-AuNPs@SH-Si was used for Ag nanoshell formation according to the previously developed method (see the Supporting Information). ${ }^{26,27}$ The treated chips were then rinsed with water and dried under a stream of $\mathrm{N}_{2}$ prior to SERS measurement.

2.6. Analysis of Active Ricin in Real Samples. Analyses of active ricin were also performed in drinking water, fresh apple juice, and human serum, which were diluted 50 times with phosphate-buffered saline (PBS) buffer before analysis. These practical samples were spiked with active ricin at various concentrations. In a typical process, the sample solution $(10 \mu \mathrm{L})$ was mixed with ammonium acetate buffer (40 $\mu \mathrm{L}, 10 \mathrm{mmol} \mathrm{L}^{-1}, \mathrm{pH} 4.0$ ) in a 96-well plate, which contained the SERS chip, and then reacted for $50 \mathrm{~min}$. After Ag deposition on the post-reacted chip, SERS measurement was conducted. Finally, the recoveries of the method were calculated and listed in Table 1.

Table 1. Determination of Active Ricin Spiked in Different Matrices by the Proposed SERS Chip

\begin{tabular}{lccc}
\multicolumn{1}{c}{ sample } & $\begin{array}{c}\text { spiked } \\
\left(\mathrm{ng} \mathrm{mL}^{-1}\right)\end{array}$ & $\begin{array}{c}\text { detected } \\
\left(\mathrm{ng} \mathrm{mL}^{-1}\right)\end{array}$ & $\begin{array}{c}\text { recovery } \\
(\%)\end{array}$ \\
drinking water & 100.0 & 99.2 & 99.2 \\
apple juice & 300.0 & 303 & 101 \\
diluted human serum & 100.0 & 98.3 & 98.3 \\
& 300.0 & 306 & 102 \\
& 100.0 & 91.7 & 91.7 \\
& 300.0 & 279 & 93.1
\end{tabular}

\section{RESULTS AND DISCUSSION}

\subsection{Preparation and Characterization of the ssODN-} AuNP Conjugate. In this assay, intact ricin was used but not individual ricin A or B chain, because only intact ricin could enter into cells and then play its toxicity. Besides, part of toxicity may be lost as a result of inactivation during the process to prepare A chain from intact ricin. On the basis of the inconsistent observations in the foregoing studies, ${ }^{28,29}$ several different lengths and base compositions of ssODN, which contain the specific sequence motif for ricin depurination, were rescreened for obtaining the optimal substrate (Figure S1 and Table S1 of the Supporting Information). It is found that the group of polydeoxyadenosine acids $[p o l y(d A)]$ with different degrees of polymerization show much higher activity than others under $\mathrm{pH} 4.0$ (Figure S2 of the Supporting Information).

Next, we fabricated ssODN-modified AuNPs and anticipated that they would amplify recognition and reaction by virtue of their cooperative binding property. To achieve this, uniform citrate-AuNPs $(\sim 100 \mathrm{~nm})$ were prepared via a kinetically controlled seeded growth strategy, followed by functionalization with ssODN. We chose the most active substrate, i.e., poly $(15 \mathrm{dA})$, for this goal. Considering the steric hindrance between poly (15dA) and AuNPs, a six-base adenine-linking spacer was added at the $5^{\prime}$ end of poly $(15 \mathrm{dA}),{ }^{30}$ which was denominated as SH-poly(21dA). As shown in Figure 1, both
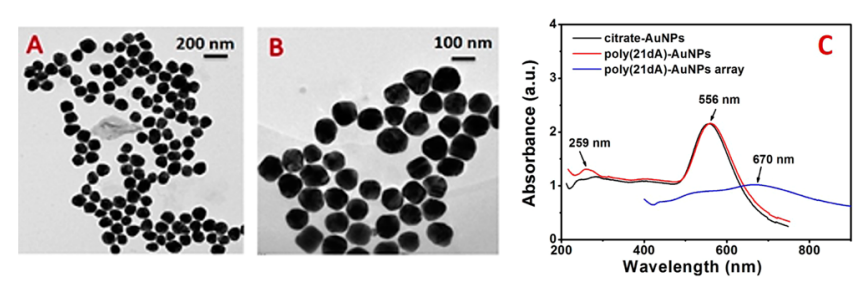

Figure 1. TEM images of (A) citrate-AuNPs and (B) poly(21dA)AuNPs and (C) UV-vis extinction spectra of the isolated AuNPs in aqueous solution and the poly(21)-AuNPs array coated with the Ag nanoshell on $\mathrm{SH}-\mathrm{Si}$.

citrate-AuNPs and poly(21dA)-AuNPs are monodispersed and display uniform spherical morphologies with size distributions of $\sim 100 \mathrm{~nm}$. As measured by the UV-vis spectroscopy (Figure 1C), the surface plasmon resonance (SPR) peak of poly $(21 \mathrm{dA})-\operatorname{AuNPs}(556 \mathrm{~nm})$ is retained but shows a slight red shift of $3 \mathrm{~nm}$ compared to that of citrateAuNPs. Simultaneously, a small additional peak appears at 259 $\mathrm{nm}$, implying the presence of ssODN. The average hydrodynamic size of citrate-AuNPs was measured as $112 \mathrm{~nm}$ with a polydispersity index (PDI) value of 0.119. After modification, the average hydrodynamic size of poly(21dA)-AuNPs increases to $116 \mathrm{~nm}$ with a PDI value of 0.127 , further indicating the presence of the poly $(21 \mathrm{dA})$ layer on AuNPs. These results suggest that the modification of poly $(21 \mathrm{dA})$ does not affect the morphology and monodispersed state of AuNPs.

Subsequently, we investigated the depurination reaction between active ricin and poly $(21 \mathrm{dA})-$ AuNPs in homogeneous solution by monitoring the amount of released adenine using liquid chromatography-mass spectrometry (LC-MS). It is found that the obtained poly $(21 \mathrm{dA})$-AuNPs show weak interaction with active ricin, reflected by the fact that far less adenine is released from poly $(21 \mathrm{dA})$-AuNPs (Figure S3 of the Supporting Information). It is speculated that $\mathrm{SH}-$ poly(21dA) 
tend to wrap and coordinate with AuNPs in a horizontally aligned conformation through the functional groups (e.g., amines and carbonyls) of adenine, thus losing its recognition and reaction ability toward active ricin. To solve this problem, ligand exchange by $\mathrm{MHO}$ was adopted to replace adenine bound on AuNPs. ${ }^{30}$ After MHO treatment, poly(21dA)AuNPs exhibit the uniform average hydrodynamic size of ca. $124 \mathrm{~nm}$, which implies that poly(21dA) discard the flat on orientation and adopt a more extended molecular conformation, which would result in the increase of the effective size. As evident by LC-MS measurement (Figure S3 of the Supporting Information), MHO-treated poly(21dA)-AuNPs show greatly improved reaction capacity with active ricin (see detailed discussion in the Supporting Information).

3.2. Morphological Characterization of the SERS Chip. Benefiting from the unique composition of poly(21dA), they are very suitable for simultaneously acting as both a Raman reporter $^{31,32}$ and a specific recognition element for active ricin in the SERS-based sensor. When all of these advantages offered by SERS are combined, an ex situ technique for depositing metal NPs with a well-defined shape on support materials is an attractive and promising way to fabricate flexible SERS sensors. ${ }^{33,34}$ In this method, we adopted a chemical method to modify the DHLA-terminated monolayer on the Si wafer $(\mathrm{SH}-\mathrm{Si})$, which could greatly enhance the binding stability of poly $(21 \mathrm{dA})-\mathrm{AuNPs}$ on $\mathrm{SH}-\mathrm{Si}$. The scanning electron microscopy (SEM) image (Figure 2A) of the SERS chip and
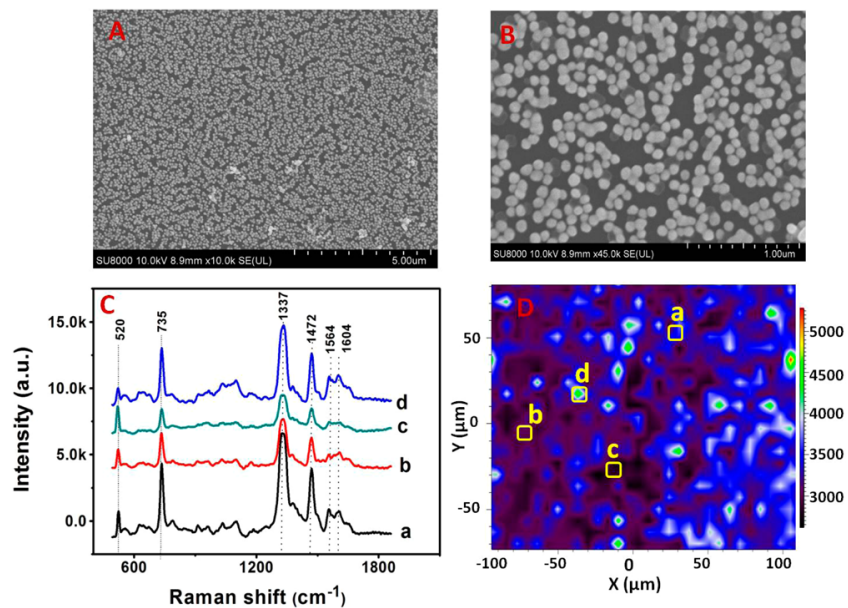

Figure 2. Characterizations for the SERS chip. (A) SEM image of poly(21dA)-AuNPs@SH-Si. (B) Close-up SEM image of the same sample. (C) SERS spectra obtained from four random regions marked in image D. (D) Raman intensity mapping of the $735 \mathrm{~cm}^{-1}$ band under laser excitation at $633 \mathrm{~nm}$.

its close-up counterpart (Figure 2B) illustrate the dense surface coverage and uniform distribution of poly $(21 \mathrm{dA})-\mathrm{AuNPs}$ on $\mathrm{SH}-\mathrm{Si}$. It is also demonstrated that there is no evident change in the poly(21dA)-AuNP dimension and shape upon transitioning from the solution phase (Figure 1B) to the surface-bound state (Figure 2B). As shown in Figure 2C, the SERS spectra from different locations of the SERS chip are very similar; i.e., all of them provide an overwhelming dominance of adenine modes, including the ring-breathing vibration at 735 $\mathrm{cm}^{-1}$ and the bond stretching at $1337 \mathrm{~cm}^{-1}$, as well as the moderately intense plane vibrations at 1472 and $1564 \mathrm{~cm}^{-1} .35$ However, they are absent in the characteristic peaks from phosphate and sugar moieties on ssODN as a result of the small
Raman cross-section of them. ${ }^{36}$ Moreover, we also evaluated the signal performance of the SERS chip fabricated with different sizes of poly(21dA)-AuNPs $(\sim 60, \sim 75, \sim 90$, and $\sim 100 \mathrm{~nm}$ ). It is found that the SERS chip fabricated with poly(21dA)-AuNPs of $\sim 100 \mathrm{~nm}$ possesses the most sensitive SERS signal (Figure S5 of the Supporting Information), which is optimal for "turn-off" SERS detection.

To evaluate the spatial homogeneity of the SERS signal, point-by-point Raman mapping was performed on a randomly selected $200 \times 150 \mu \mathrm{m}^{2}$ area on poly(21dA)-AuNPs@SH-Si. Identical color intensities of the mapping image show a homogeneous SERS signal over a large area (Figure 2D), with a corresponding relative standard deviation (RSD) of less than $10 \%$. Under the same test conditions, the coefficient of variation for SERS performance was calculated at less than $15 \%$ for the same batch. Similarly, substrate-substrate spectral reproducibility was also analyzed, and a coefficient of variation below $20 \%$ was obtained for five different batches, which demonstrate the generation of SERS signals with good homogeneity and reproducibility.

3.3. Specific Detection of Active Ricin Using Poly(21dA)-Au@SH-Si. As the concentration of ricin increases, the overall spectral intensities are rather quantitatively decreased with similar spectral features (Figure 3), even though

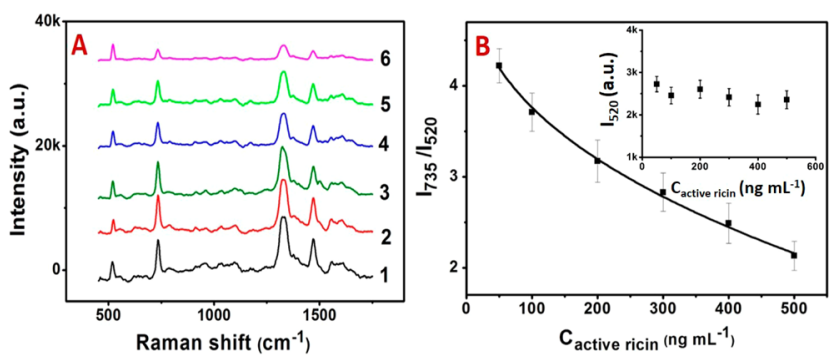

Figure 3. (A) Representative SERS spectra of poly(21dA)-AuNPs@ $\mathrm{SH}-\mathrm{Si}$ treated with the increasing concentrations of active ricin from 1 to 6: $50,100,200,300,400$, and $500 \mathrm{ng} \mathrm{mL}^{-1}$. All of SERS spectra are the average results of 50 spots randomly distributed on the SERS chip. (B) Plot of $I_{735} / I_{520}$ as a function of the active ricin concentration. All error bars represent standard deviation $(n=5)$.

the depurination of adenine on poly(21dA)-Au@SH-Si is random. As a comparison, MHO-AuNPs@SH-Si was prepared and evaluated its interaction with active ricin. It is found that MHO-AuNPs@SH-Si show a low SERS signal as a result of the small Raman cross-section of MHO. After incubation with ricin, no distinct SERS band appeared, which reflects weak interaction of MHO-AuNPs@SH-Si with ricin as a result of the hydrophobic and neutral property of $\mathrm{MHO}$ (Figure S6 of the Supporting Information). Quantification of active ricin was conducted by plotting the relative SERS intensities at $735 \mathrm{~cm}^{-1}$ compared to the Raman signal at 520 $\mathrm{cm}^{-1}\left(I_{735} / I_{520}\right)$ against the active ricin concentration $\left(C_{\text {active ricin }}\right)$, as shown in Figure 3B. Raman signals at 520 $\mathrm{cm}^{-1}$ from $\mathrm{Si}$ show intensity variability (inset of Figure $3 \mathrm{~B}$ ) because the coverage rate of the AuNP array on the Si substrate is not exactly identical to each piece of the chip. It is found that $I_{735} / I_{520}$ versus the active ricin concentration shows a power law relationship correlation, $I_{735} / I_{520}=5.58-0.296 C_{\text {active ricin }}{ }^{0.393}$, in the range of $\left.50-500 \mathrm{ng} \mathrm{mL}-1 R^{2}=0.996\right)$. The limit of detection (LOD), defined as the analyte concentration equivalent to three standard deviations of 11 repeated 
measurements of a blank solution $(3 \sigma)$, is $47.5 \mathrm{ng} \mathrm{mL}^{-1}$. Here, the peak of $735 \mathrm{~cm}^{-1}$ assigned to the ring-breathing vibration of adenine was selected to represent the SERS response because it exhibits an intensive signal and a minimum standard deviation in the sensing process. Time-dependent alteration of the SERS signal after treatment with active ricin was investigated (Figure S7 of the Supporting Information). It is found that the signal decreased within the initial $60 \mathrm{~min}$ and then leveled off. Therefore, a reaction time of $60 \mathrm{~min}$ was chosen to ensure complete reaction. Besides, we adopted a simple silver-staining methodology ${ }^{26,27}$ for gradually coating poly(21dA)-AuNPs@ $\mathrm{SH}-\mathrm{Si}$ with the Ag nanoshell. The maximum signal intensity of the SERS chip was obtained after Ag growth for $40 \mathrm{~min}$, which is approximately 5.5 orders of magnitude higher than that of the uncoated counterpart (Figure S6 of the Supporting Information). With prolonged growth time, the signal intensity starts to level off. These results suggest that incomplete filling of the Ag nanoshell induces nanopinhole formation on the AuNP surface. As the Ag nanoshell increases in thickness, more reporter molecules may be embedded but it would be more difficult for photons to reach these reporters and then escape. ${ }^{37,38}$ Therefore, the combination of these two effects makes an intermediate thickness of the $\mathrm{Ag}$ nanoshell the most favorable for high sensitivity. The as-fabricated poly $(21 \mathrm{dA})-\mathrm{AuNP}$ array after $\mathrm{Ag}$ deposition displays a red-shifted broad plasmon band at $670 \mathrm{~nm}$ (Figure 1C). Therefore, a $633 \mathrm{~nm}$ laser was selected as the excitation wavelength of the SERS spectra in this assay.

3.4. Analytical Performance of the SERS Chip. Figure 4 reveals the SERS signal attenuation with increasing concen-
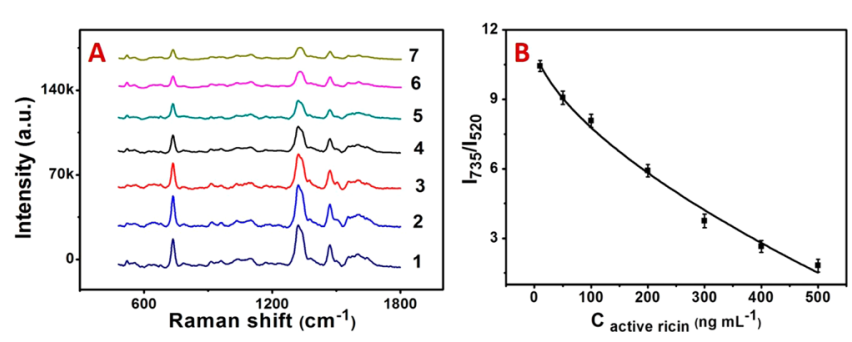

Figure 4. (A) Typical SERS spectra with respect to different concentrations of active ricin: $0,10,100,200,300,400$, and $500 \mathrm{ng}$ $\mathrm{mL}^{-1}$ from 1 to 7 for post-reacted poly(21dA)-AuNPs@SH-Si after $\mathrm{Ag}$ nanoshell deposition. All of SERS spectra are the average results of 50 spots randomly distributed on the SERS chip. (B) Calibration curve for the determination of the active ricin concentration. All error bar represent standard deviation $(n=5)$.

trations of active ricin, and $I_{735} / I_{520}$ versus the active ricin concentration also shows a power law relationship correlation in the range of $10-500 \mathrm{ng} \mathrm{mL} L^{-1}$. The regression equation is $I_{735} / I_{520}=11.36-0.198 C_{\text {active ricin }}^{0.623}\left(R^{2}=0.991\right)$, and the LOD $(3 \sigma)$ is $8.9 \mathrm{ng} \mathrm{mL}^{-1}$. Previous research showed that the median lethal dose $\left(\mathrm{LD}_{50}\right)$ for ricin in humans is around 5-10 $\mu \mathrm{g} \mathrm{kg}^{-1}$ of body weight (BW) through injection or inhalation and $1-20 \mathrm{mg} \mathrm{kg}^{-1}$ of BW or 8 castor beans through ingestion. On the basis of the toxicity data, the LOD of our assay is low enough for risk assessment. ${ }^{39-41}$ If the concentration of active ricin in real samples is higher than the upper limit of the linear range, this assay can first provide warning information. Then, for accurate quantitation, the samples need to be diluted to fall into the linear range of the assay.

The reproducibility of the method was examined by six parallel measurements at a concentration of $200 \mathrm{ng} \mathrm{mL}^{-1}$ ricin on different substrates, which show a RSD of $4.2 \%$. It was found that the SERS chip possesses high specificity for active ricin. Neither common proteases (including lysozyme, pronase, and trypsin), PNGase $\mathrm{F}$, nor $\beta$-GUS affected the SERS signals at concentrations of $1.0 \mathrm{mg} \mathrm{mL}{ }^{-1}$ (Figure 5). Actually, our

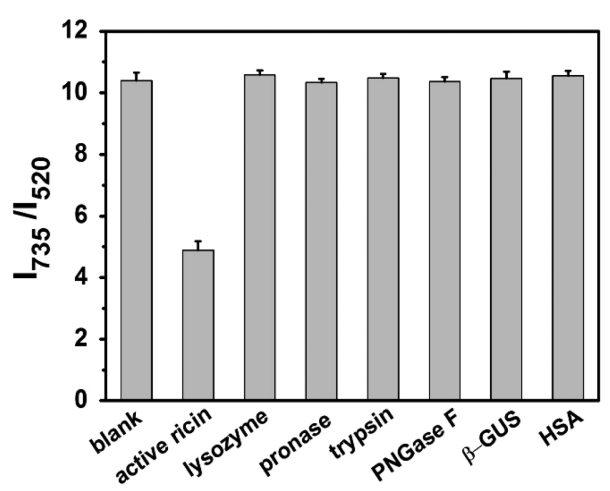

Figure 5. Selectivity of the SERS chip in the presence of various proteins (active ricin, $250 \mathrm{ng} \mathrm{mL}^{-1}$; other proteins, $1.0 \mathrm{mg} \mathrm{mL}^{-1}$ ). All error bar represent standard deviation $(n=5)$.

established assay can be used to test the toxicity of all type II ribosome-inactivating proteins (RIP II), which could hydrolyze this synthetic poly(dA21) substrate to release adenine. Among RIP II, ricin is the most representative toxin, with an extreme threat to the security of the public, which makes the proposed SERS chip have great realistic significance.

3.5. Analysis of Active Ricin in Complex Matrices. Ricin is a very toxic protein and a potential bioterrorism threat. To evaluate the feasibility of the proposed SERS chip used in real samples, active ricin was detected in various spiked food and biological samples. It is found that the SERS spectra show less interference from complex samples, because the sensing interface of the SERS chip was pre-covered with $\mathrm{SH}-$ poly (21dA) and $\mathrm{MHO}$ via a strong $\mathrm{Au}-\mathrm{S}$ interaction, which could greatly block AuNPs and, thus, reduce chemi-adsorption of impurities from real samples on the AuNP array (Figure S9 of the Supporting Information). According to the calibration curve obtained from the buffer system, acceptable results with relative errors of less than $7.6 \%$ and good recoveries between 91.7 and $102 \%$ were obtained (Table 1). Benefiting from wellprotected ssODNs linked on AuNPs against degradation, the SERS chip possesses better stability. It is found that, after storage under $4{ }^{\circ} \mathrm{C}$ for about 3 month, the SERS spectra of the chip were kept almost unchanged (Figure S10 of the Supporting Information), which make it possible to prepare the SERS chip prior to analysis and store them until they are needed.

\section{CONCLUSION}

In this work, a series of ssODNs were examined, and the optimal substrate for in vitro ricin depurination under specific conditions was selected. Using this ssODN, we developed a SERS chip that combines plasmonic and specific sensing capacities in one entity. The design of the SERS chip presents an open surface topology amenable to not only easily incorporating specific chemical or biological moieties but also facilitating target recognition and mass transfer. Meanwhile, the large-area uniformity was very helpful for the generation of reproducible SERS spectra. Furthermore, Ag deposition on the post-reacted SERS chip afforded synergistically increased SERS 
signals as a result of the constructive enhancement of electromagnetic (EM) fields at "hot spots". Benefiting from its readily acquired, minimum-cost, one-step quantitation compared to previously reported methods (Table S2 of the Supporting Information), this method offers distinct advantages for rapid on-site detection of active ricin.

\section{ASSOCIATED CONTENT}

\section{S Supporting Information}

The Supporting Information is available free of charge on the ACS Publications website at DOI: 10.1021/acsami.5b12860.

Experimental details for liquid chromatography-electrospray ionization-time-of-flight/mass spectrometry (LCESI-TOF/MS) experiments, SERS measurement condition, SERS signal processing, synthesis and characterization of $\mathrm{SH}-\mathrm{Si}$ and $\mathrm{MHO}-\mathrm{AuNPs} @ \mathrm{SH}-\mathrm{Si}$, modification of the selected depurination substrate to AuNPs, deposition of the Ag nanoshell on poly(21dA)AuNPs@SH-Si, detection of active ricin in spiked samples, stability of poly(dA21)-AuNPs@SH-Si, extra figures and tables, and deep interpretation of these data (PDF)

\section{AUTHOR INFORMATION}

\section{Corresponding Authors}

*E-mail: jfliu@rcees.ac.cn.

*E-mail: xiejw@bmi.ac.cn.

\section{Author Contributions}

${ }^{\dagger} \mathrm{Ji}$-jun Tang and Jie-fang Sun contributed equally to this work. Notes

The authors declare no competing financial interest.

\section{ACKNOWLEDGMENTS}

This work was supported by the Strategic Priority Research Program of the Chinese Academy of Sciences (XDB14020101), the National Major Instrument Project of the Ministry of Science and Technology of China (No. 2011YQ03012411), the National Natural Science Foundation of China (21207142, 21321004, and 81202248), and the China Postdoctoral Science Foundation (2012M510048).

\section{REFERENCES}

(1) Lord, J. M.; Roberts, L. M.; Robertus, J. D. Ricin: Structure, Mode of Action, and Some Current Applications. FASEB J. 1994, 8, 201-208.

(2) Brandon, D. L. Detection of Ricin Contamination in Ground Beef by Electrochemiluminescence Immunosorbent Assay. Toxins 2011, 3, 398-408.

(3) Kalb, S. R.; Barr, J. R. Mass Spectrometric Detection of Ricin and its Activity in Food and Clinical Samples. Anal. Chem. 2009, 81, 20372042.

(4) McGrath, S. C.; Schieltz, D. M.; McWilliams, L. G.; Pirkle, J. L.; Barr, J. R. Detection and Quantification of Ricin in Beverages Using Isotope Dilution Tandem Mass Spectrometry. Anal. Chem. 2011, 83, 2897-2905.

(5) Wang, B.; Guo, C.; Chen, G.; Park, B.; Xu, B. Following Aptamer-Ricin Specific Binding by Single Molecule Recognition and Force Spectroscopy Measurements. Chem. Commun. 2012, 48, 16441646.

(6) Lamont, E. A.; He, L.; Warriner, K.; Labuza, T. P.; Sreevatsan, S. A Single DNA Aptamer Functions as a Biosensor for Ricin. Analyst 2011, 136, 3884-3895.
(7) He, L.; Lamont, E.; Veeregowda, B.; Sreevatsan, S.; Haynes, C. L.; Diez-Gonzalez, F.; Labuza, T. P. Aptamer-based Surface-enhanced Raman Scattering Detection of Ricin in Liquid Foods. Chem. Sci. 2011, 2, 1579-1582.

(8) Rasooly, R.; He, X. Sensitive Bioassay for Detection of Biologically Active Ricin in Food. J. Food Prot. 2012, 75, 951-954.

(9) Rastogi, V. K.; Wallace, L. Novel Cell-Based Assay for Testing Active Holo-Ricin and Its Application in Detection Following Decontamination; United States Environmental Protection Agency (U.S. EPA): Washington, D.C., 2010; EPA 600/R-10/097.

(10) Sturm, M. B.; Schramm, V. L. Detecting Ricin: Sensitive Luminescent Assay for Ricin A Chain Ribosome Depurination Kinetics. Anal. Chem. 2009, 81, 2847-2853.

(11) Bevilacqua, V. L.; Nilles, J. M.; Rice, J. S.; Connell, T. R.; Schenning, A. M.; Reilly, L. M.; Durst, H. D. Ricin Activity Assay by Direct Analysis in Real Time Mass Spectrometry Detection of Adenine Release. Anal. Chem. 2010, 82, 798-800.

(12) Becher, F.; Duriez, E.; Volland, H.; Tabet, J. C.; Ezan, E. Detection of Functional Ricin by Immunoaffinity and Liquid Chromatography-Tandem Mass Spectrometry. Anal. Chem. 2007, 79, 659-665.

(13) Halvorson, R. A.; Vikesland, P. J. Surface-Enhanced Raman Spectroscopy (SERS) for Environmental Analyses. Environ. Sci. Technol. 2010, 44, 7749-7755.

(14) Yap, F. L.; Thoniyot, P.; Krishnan, S.; Krishnamoorthy, S. Nanoparticle Cluster Arrays for High-Performance SERS through Directed Self-Assembly on Flat Substrates and on Optical Fibers. ACS Nano 2012, 6, 2056-2070.

(15) Kneipp, J.; Kneipp, H.; McLaughlin, M.; Brown, D.; Kneipp, K. In Vivo Molecular Probing of Cellular Compartments with Gold Nanoparticles and Nanoaggregates. Nano Lett. 2006, 6, 2225-2231.

(16) Liu, B.; Han, G.; Zhang, Z.; Liu, R.; Jiang, C.; Wang, S.; Han, M. Shell Thickness-Dependent Raman Enhancement for Rapid Identification and Detection of Pesticide Residues at Fruit Peels. Anal. Chem. 2012, 84, 255-261.

(17) Xu, J.; Turner, J. W.; Idso, M.; Biryukov, S. V.; Rognstad, L.; Gong, H.; Trainer, V. L.; Wells, M. L.; Strom, M. S.; Yu, Q. In Situ Strain-Level Detection and Identification of Vibrio parahaemolyticus Using Surface-Enhanced Raman Spectroscopy. Anal. Chem. 2013, 85, 2630-2637.

(18) Piorek, B. D.; Lee, S. J.; Moskovits, M.; Meinhart, C. D. FreeSurface Microfluidics/Surface-enhanced Raman Spectroscopy for RealTime Trace Vapor Detection of Explosives. Anal. Chem. 2012, 84, 9700-9705.

(19) Zhang, X.; Zhao, J.; Whitney, A. V.; Elam, J. W.; Van Duyne, R. P. Ultrastable Substrates for Surface-Enhanced Raman spectroscopy: $\mathrm{Al}_{2} \mathrm{O}_{3}$ Overlayers Fabricated by Atomic Layer Deposition Yield Improved Anthrax Biomarker Detection. J. Am. Chem. Soc. 2006, 128, 10304-10309.

(20) Golightly, R. S.; Doering, W. E.; Natan, M. J. Surface-Enhanced Raman Spectroscopy and Homeland Security: A Perfect Match? ACS Nano 2009, 3, 2859-2869.

(21) Algar, W. R.; Krull, U. J. Multidentate Surface Ligand Exchange for the Immobilization of $\mathrm{CdSe} / \mathrm{ZnS}$ Quantum Dots and Surface Quantum Dot-Oligonucleotide Conjugates. Langmuir 2008, 24, $5514-5520$.

(22) Stokes, R. J.; Macaskill, A.; Lundahl, P. J.; Smith, W. E.; Faulds, K.; Graham, D. Quantitative Enhanced Raman Scattering of Labeled DNA from Gold and Silver Nanoparticles. Small 2007, 3, 1593-1601.

(23) Tang, J.; Xie, J.; Shao, N.; Yan, Y. The DNA Aptamers That Specifically Recognize Ricin Toxin are Selected by Two In Vitro Selection Methods. Electrophoresis 2006, 27, 1303-1311.

(24) Bastus, N. G.; Comenge, J.; Puntes, V. Kinetically Controlled Seeded Growth Synthesis of Citrate-Stabilized Gold Nanoparticles of up to 200 nm: Size Focusing versus Ostwald Ripening. Langmuir 2011, 27, 11098-11105.

(25) Zhang, X.; Servos, M. R.; Liu, J. Instantaneous and Quantitative Functionalization of Gold Nanoparticles with Thiolated DNA Using a 
pH-Assisted and Surfactant Free Route. J. Am. Chem. Soc. 2012, 134, $7266-7269$.

(26) Lee, J. H.; Kim, G. H.; Nam, J. M. Directional Synthesis and Assembly of Bimetallic Nanosnowmen with DNA. J. Am. Chem. Soc. 2012, 134, 5456-5459.

(27) Zhang, Z.; Zhang, S.; Lin, M. DNA-Embedded Au-Ag CoreShell Nanoparticles Assembled on Silicon Slides as a Reliable SERS Substrate. Analyst 2014, 139, 2207-2213.

(28) Lord, M. J.; Jolliffe, N. A.; Marsden, C. J.; Pateman, C. S.; Smith, D. C.; Spooner, R. A.; Watson, P. D.; Roberts, L. M. Ricin: Mechanisms of Cytotoxicity. Toxicol. Rev. 2003, 22, 53-64.

(29) Barbieri, L.; Valbonesi, P.; Bonora, E.; Gorini, P.; Bolognesi, A.; Stirpe, F. Polynucleotide: Adenosine Glycosidase Activity of Ribosome-Inactivating Poteins: Effect on DNA, RNA and poly(A). Nucleic Acids Res. 1997, 25, 518-522.

(30) Demers, L. M.; Östblom, M.; Zhang, H.; Jang, N. H.; Liedberg, B.; Mirkin, C. A. Thermal Desorption Behavior and Binding Properties of DNA Bases and Nucleosides on Gold. J. Am. Chem. Soc. 2002, 124, 11248-11249.

(31) Li, H.; Sun, J.; Cullum, B. M. Label-Free Detection of Proteins Using SERS-Based Immune-Nanosensors. NanoBiotechnology 2006, 2, $17-28$.

(32) Bell, S. E. J.; Sirimuthu, N. M. S. Surface-Enhanced Raman Spectroscopy (SERS) for Sub-Micromolar Detection of DNA/RNA Mononucleotides. J. Am. Chem. Soc. 2006, 128, 15580-15581.

(33) Braun, G.; Lee, S. J.; Dante, M.; Nguyen, T. Q.; Moskovits, M.; Reich, N. Surface-Enhanced Raman Spectroscopy for DNA Detection by Nanoparticle Assembly onto Smooth Metal Films. J. Am. Chem. Soc. 2007, 129, 6378-6379.

(34) Freeman, R. G.; Grabar, K. C.; Allison, K. J.; Bright, R. M.; Davis, J. A.; Guthrie, A. P.; Hommer, M. B.; Jackson, M. A.; Smith, P. C.; Walter, D. G.; Natan, M. J. Self-Assembled Metal Colloid Monolayers: An Approach to SERS Substrates. Science 1995, 267, 1629-1632.

(35) Deng, H.; Bloomfield, V. A.; Benevides, J. M.; Thomas, J. G. Dependence of the Raman Signature of Genomic B-DNA on Nucleotide Base Sequence. Biopolymers 1999, 50, 656-666.

(36) Kundu, J.; Neumann, O.; Janesko, B. G.; Zhang, D.; Lal, S.; Barhoumi, A.; Scuseria, G. E.; Halas, N. J. Adenine-and Adenosine Monophosphate (AMP)-Gold Binding Interactions Studied by Surface-Enhanced Raman and Infrared Spectroscopies. J. Phys. Chem. C 2009, 113, 14390-14397.

(37) Lim, D. K.; Jeon, K. S.; Kim, H. M.; Nam, J. M.; Suh, Y. D. Nanogap-Engineerable Raman-Active Nanodumbbells for SingleMolecule Detection. Nat. Mater. 2010, 9, 60-67.

(38) Lee, H.; Lee, J. H.; Jin, S. M.; Suh, Y. D.; Nam, J. M. SingleMolecule and Single-Particle-Based Correlation Studies between Localized Surface Plasmons of Dimeric Nanostructures with $\sim 1 \mathrm{~nm}$ Gap and Surface-Enhanced Raman Scattering. Nano Lett. 2013, 13, 6113-6121.

(39) Balint, G. A. Ricin: the toxic protein of castor oil seeds. Toxicology 1974, 2, 77-102.

(40) Bradberry, S. M.; Dickers, K. J.; Rice, P.; Griffiths, G. D.; Vale, J. A. Ricin Poisoning. Toxicol. Rev. 2003, 22, 65-70.

(41) Wu, X.; Gao, S.; Wang, J. S.; Wang, H.; Huang, Y. W.; Zhao, Y. The Surface-Enhanced Raman Spectra of Aflatoxins: Spectral Analysis, Density Functional Theory Calculation, Detection and Differentiation. Analyst 2012, 137, 4226-4234. 